\title{
Mitogen-activated protein kinase activator with WD40 repeats (MAWD) and MAWD- binding protein induce cell differentiation in gastric cancer
}

Dongmei Li ${ }^{1}$, Jun Zhang ${ }^{2,3}$, Yu Xi ${ }^{4}$, Lei Zhang ${ }^{5}$, Wenmei Li ${ }^{2}$, Jiantao Cui ${ }^{2}$, Rui Xing ${ }^{2}$, Yuanmin Pan ${ }^{2}$, Zemin Pan ${ }^{1}$, Feng $\mathrm{Li}^{1}$ and Youyong $\mathrm{Lu}^{2^{*}}$

\begin{abstract}
Background: Our previous proteomic analysis revealed that mitogen-activated protein kinase activator with WD40 repeats (MAWD) and MAWD-binding protein (MAWBP) were downregulated in gastric cancer (GC) tissues. These proteins interacted and formed complexes in GC cells. To investigate the role of MAWD and MAWBP in GC differentiation, we analyzed the relationship between MAWD/MAWBP and clinicopathologic characteristics of GC tissues and examined the expression of E-cadherin and pepsinogen C (PGC)—used as gastric mucosa differentiation markers -in MAWD/MAWBP-overexpressing GC cells and xenografts.

Methods: We measured MAWD, MAWBP, transforming growth factor-beta (TGF-beta), E-cadherin, and PGC expression in $223 \mathrm{GC}$ tissues and matched-adjacent normal tissues using tissue microarray and immunohistochemistry (IHC) analyses, and correlated these expression levels with clinicopathologic features. MAWD and MAWBP were overexpressed alone or together in SGC7901 cells and then E-cadherin, N-cadherin, PGC, Snail, and p-Smad2 levels were determined using western blotting, semiquantitative RT-PCR, and immunofluorescence analysis. Alkaline phosphatase (AKP) activity was measured to investigate the differentiation level of various transfected cells, and the transfected cells were used in tumorigenicity assays and for IHC analysis of protein expression in xenografts.
\end{abstract}

Results: MAWD/MAWBP positive staining was significantly lower in GC tissues than in normal samples $(P<0.001)$, and the expression of these proteins was closely correlated with GC differentiation grade. Kaplan-Meier survival curves indicated that low MAWD and MAWBP expression was associated with poor patient survival $(P<0.05)$. The differentiation-related proteins E-cadherin and PGC were expressed in GC tissues at a lower level than in normal tissues $(P<0.001)$, but were upregulated in MAWD/MAWBP-overexpressing cells. N-cadherin and Snail expression was strongr in vector-expressing cells and comparatively weaker in MAWD/MAWBP co-overexpressing cells. MAWD/MAWBP Co-overexpression inhibited Smad2 phosphorylation and nuclear translocation $(P<0.05)$, and AKP activity was lowest in MAWD/MAWBP coexpressing cells and highest in vector-expressing cells $(P<0.001)$. TGF-beta, E-cadherin, and PGC expression in xenograft tumors derived from MAWD/MAWBP coexpressing cells was higher than that in control.

Conclusions: MAWD and MAWBP were downregulated and associated with the differentiation grade in GC tissues. MAWD and MAWBP might induce the expression of differentiation-related proteins by modulating TGF-beta signaling in GC cells.

\footnotetext{
* Correspondence: 10989959@bjmu.edu.cn

${ }^{2}$ Laboratory of Molecular Oncology, Key Laboratory of Carcinogenesis and

Translational Research (Ministry of Education), Peking University Cancer

Hospital/Institute, Beijing 100142, P.R China

Full list of author information is available at the end of the article
} 


\section{Background}

Gastric cancer (GC) is one of the most common malignancies worldwide and ranks second in terms of global cancer-related mortality [1]. Host genetic factors as well as bacterial virulence, environmental, and several other factors have been shown to affect the gastric oncogenic process, but the underlying molecular mechanism is poorly understood.

GC displays distinct biological behaviors according to histological differentiation $[2,3]$, and the prognosis of GC patients is closely associated with histological classification: The 5-year survival rates of GC patients are $90 \%$, $50 \%-60 \%$, and $10 \%-15 \%$ for GC Stages I, II, and III, respectively [4]. Thus, it is critical to elucidate the regulatory mechanism of GC cell differentiation, and previous studies have investigated the mechanism of induced differentiation in GC cells. Sakamoto et al. determined that in addition to intestinal transcription factor caudal type homeobox 2, epidermal growth factor receptor (EGFR) activation induces LI-cadherin expression and participates in the intestinal differentiation in GC [5]. Wei et al. reported that P27 regulation by glycogen synthase kinase-3beta results in hexamethylene bisacetamideinduced differentiation of human GC cells [6]. Hsu et al. found that the loss of RUNX3 expression correlates with GC differentiation [7]. However, few reports have been published on proteins related to the differentiation and proliferation of GC cells.

Previously, we determined-using 2D gel electrophoresis and mass spectrometry-that the expression of mitogen-activated protein kinase activator with WD40 repeats (MAWD) and MAWD-binding protein (MAWBP) was markedly attenuated in GC tissues. These proteins interacted and formed complexes in GC cells, and this might play a major role in GC carcinogenesis [8].

The effects of MAWD in cancers have been described in a few reports. MAWD is evolutionarily conserved and expressed in diverse tissues $[9,10]$. Iriyama and colleagues attempted to detect MAWD-related proteins by using the conventional two-hybrid technique and found that MAWBP can bind to MAWD [10]. Buess et al. reported complete or partial allelic loss of MAWD in $45.2 \%(75 / 166)$ of colorectal cancers [11]. Jung et al. found that MAWD bound to NM23-H1 and that this created a complex that interacted with, and potentiated the activity of, p53 [12]. Dong et al. detected chromosomal deletions in prostate cancer that overlapped with the $M A W D$ location [13]. Matsuda et al. determined that MAWD was overexpressed in $45.6 \%(21 / 46)$ of human breast tumor tissues and promoted anchorage-independent cell growth [9]. Kim et al. reported MAWD upregulation in $50.8 \%$ $(30 / 59)$ of adenomas and $70.7 \%(87 / 123)$ of colorectal cancers [14]. Lastly, Halder et al. found that serinethreonine kinase receptor-associated protein, or STRAP, was upregulated in $60 \%(12 / 20)$ of colon and $78 \%(11 / 14)$ of lung carcinomas [15]. However, no reports have been published on the function of MAWD in GC, and little is known about MAWBP other than that it can interact with MAWD.

MAWD, as the name suggests, contains a WD40 repeat domain [16]. Datta et al. showed that MAWD recruits Smad7 and forms a complex that increases the inhibition of transforming growth factor-beta (TGFbeta) signaling $[17,18]$. We hypothesized that MAWD and MAWBP interactions play a key role in the differentiation of GC. Therefore, we investigated the relationship between the expression of MAWD/MAWBP and the differentiation grade of $\mathrm{GC}$ by using clinical samples, and we also examined the expression of differentiation-related proteins in MAWD/MAWBPoverexpressing GC cells and xenografts. Lastly, we determined whether MAWD and MAWBP induce differentiation through TGF-beta signaling in GC. Research on proteins that influence the differentiation of GC will not only contribute to the diagnosis of GC: it will also help guide $\mathrm{GC}$ treatment.

\section{Methods}

\section{Sample collection}

Clinical data and GC samples were collected from Beijing Cancer Hospital of Peking University, Beijing, China, from January 2011 to June 2013. None of the patients received chemotherapy or radiotherapy before tissue samples were obtained. All histological diagnoses were confirmed by experienced pathologists at the hospital. Written informed consent was obtained from all patients regarding the use of the collected samples in research studies. The patient records and information were anonymized and de-identified before analysis. The research project and the informed consent were examined and certified by the Ethics Committee of the School of Oncology, Peking University (Beijing Cancer Hospital, China) (No. ECBCH-2011228).

\section{Immunohistochemistry (IHC) and tissue microarray (TMA)}

The gastric TMA was constructed using a tissue arraying instrument (Beecher Instruments, Silver Spring, USA), as described previously [19]. The avidin-biotinperoxidase protocol was used for IHC. The antibodies used were against MAWBP (1:100; custom-made, clone number AbM51007) and MAWD (1:300; custom-made, clone number AbP61014) [8], and TGF-beta (1:100; cat\# ab66043, Abcam, Cambridge, UK), E-cadherin (1:100; cat\# 610182, BD, Franklin, USA), and pepsinogen C (PGC) (1:150; cat\# R31924, Sigma, Cambridge, USA). Samples were incubated with antibodies at $4{ }^{\circ} \mathrm{C}$ 
overnight and visualized using the DAB kit (Dako, Glostrup, Denmark). All sections were examined and scored by 2 pathologists in a blinded evaluation. Staining was scored based on intensity and proportion. The signal intensity was scored as 0 , no staining; $1+$, low intensity; $2+$, moderate intensity; or $3+$, high intensity. The extent of surface area containing the target protein was scored on a scale of $0-3$ : (0,: no staining; $1+$ : present, but $<20 \%$; $2+: 20 \%-50 \%$; and $3+:>50 \%)$. The positivity score was calculated by multiplying staining intensity and surface area data by tissue compartment (range: 0-9), and the composite scores were separated using a four-tier system (negative: $0-1 ; 1+$ : $2-4 ; 2+$ : $5-7$; and 3+: 8-9).

\section{Prediction for potential MAWD and MAWBP protein-protein interaction (PPI) networks}

The PPI network provides an integrative view of molecular processes. The human protein interaction network was retrieved from http://www.hprd.org/; MAWDand MAWBP- interacting proteins were then searched for candidate protein-interaction sequence motifs (trimers and tetramers).

\section{Plasmid construction}

We reconstructed MAWD and MAWBP expression vectors using pcDNA3.1 B (-). Total RNA was extracted from 19-week-old fetal liver. MAWD and MAWBP CDNAs were produced using reverse-transcription PCR (RT-PCR). The MAWD primers were the following: forward: 5'-CGCGGATCCATGGCAATGAGACA GACG-3', reverse: 5'-CCCAAGCTTTCAGGCCTTAACATCAGG-3'. The amplicons were $1053 \mathrm{bp}$ in size. The MAWBP primers were the following: forward: 5'- AACTTGGTCG ACCAG CTTGCAAGGAAAATG-3', reverse: 5'-ATAACTCGAGCTAGGCTGTCAGTGT GCC-3'. The amplicons were 867 bp in size. PCR was performed as follows: the reaction was initiated using a 5 -min incubation at $94{ }^{\circ} \mathrm{C}$, and this was followed by 35 cycles of $94{ }^{\circ} \mathrm{C}$ for $45 \mathrm{~s}, 56{ }^{\circ} \mathrm{C}$ for $45 \mathrm{~s}$, and $72{ }^{\circ} \mathrm{C}$ for $60 \mathrm{~s}$, and then the reaction was terminated after a 10-min extension at $72{ }^{\circ} \mathrm{C}$. Products were purified through gel extraction, and the recombinant plasmids were transferred into Escherichia coli DH5 $\alpha$ and then identified by performing restriction-enzyme digestion and sequencing analysis.

\section{Cell culture and transfection}

The cell line SGC7901 was routinely maintained as previously described [20]. SGC7901 cells were selected and cultured at $60 \%-70 \%$ confluence in 35-mm plates and then transfected with recombinant MAWD and MAWBP plasmids or empty vector by using Lipofectamine 2000 (Invitrogen, Carlsbad, CA, USA). MAWD and MAWBP plasmids were co-transfected into SGC7901 cells, and at
$48 \mathrm{~h}$ post-transfection, the cells were seeded in selection medium containing $400 \mu \mathrm{g} / \mathrm{mL}$ G418 and cultured for 21 days to screen for stable clones.

\section{RT-PCR and western blotting}

To confirm efficient transfection, RT-PCR and western blotting were performed. Total RNA was extracted using Trizol (Invitrogen) and $5 \mu \mathrm{g}$ of the RNA was reverse transcribed and PCR-amplified. The primers used and the amplicon sizes were the following: MAWD: forward, 5'-G GGACAGGATAAACTTTAGC-3', and reverse, 5'-AGCA TGATCCCAAAGTCG AAC-3' (amplicon size, 162 bp); and MAWBP: forward, 5'-GGGTCTGCACACGCTGT TC-3', and reverse: 5'-TAATGTCAACCCTTCCGTCT-3 (132 bp). The internal control, beta-actin, was processed concurrently with all specimens. The other primers used were the following: E-cadherin: forward, 5-TGATTCTGC TGCTCTTGCTG-3', and reverse, 5'-CTCTTCTCCGCC TCCTTCTT-3' (122 bp); N-cadherin: forward, 5'-CGTG AAGGTTTGCCAGTGT-3', and reverse, 5'- CAGCACAA GGATAAGCAGGA-3' (130 bp); PGC: forward: 5'-CG TCC ACCTACTCCACCAAT-3', and reverse, 5'-CACTC AA GCCGAACTCCTG-3'(132 bp); and Snail: forward, 5'CCAGAGTTTACCTTCCAGCA G-3', and reverse, 5'-G ACA GAGTCCCAGATGAGCA-3' (214 bp). All primers were synthesized by Sangon Biotech (Shanghai) Co., Ltd. (Shanghai, China).

For western blotting, cell extracts were prepared and then the proteins $(50 \mu \mathrm{g})$ were separated on $12 \%$ SDSPAGE and transferred to PVDF membranes. The blots were stained (overnight, $4{ }^{\circ} \mathrm{C}$ ) with the following antibodies (diluted in blocking buffer): anti-MAWD (1:500), antiMAWBP (1:500), anti-Snail (1:1000; cat\# C15D3, Cell Signaling, Danvers, USA), anti-E-cadherin (1:1000), anti-Ncadherin (1:1000; cat\# 610921, BD), anti-PGC (1:1000), and anti-p-Smad2 (1:500; cat\# AB3849, Millipore, Temecula, USA). The immunoreactive bands were detected using Super Signal West Dura Extended Duration Substrate (Thermo Scientific, Rockford, USA). These experiments were repeated thrice.

\section{Immunofluorescence}

Cells were grown on glass slides, washed with PBS, methanol-fixed for $10 \mathrm{~min}$, and then processed for immunofluorescence. Cells were exposed to antibodies against E-cadherin, $\mathrm{N}$-cadherin, Snail, PGC, and p-Smad2 (all diluted 1:50) overnight at $4{ }^{\circ} \mathrm{C}$, and then incubated for $60 \mathrm{~min}$ with fluorophore-conjugated secondary antibodies; nuclei were stained with 4',6-diamidino-2-phenylindole (DAPI). Cells were examined using a Confocal Fluorescence Imaging Microscope TCS-SP5 (Leica, Mannheim, Germany). Three repeated scan results of mean fluorescence intensity were analyzed. 


\section{Alkaline phosphatase (AKP) assay}

The Alkaline Phosphatase Assay Kit (Jiancheng Bioengineering Institute, Nanjing, China) was used for measuring intracellular AKP activity. We used 3 hree holes for the detection and repeated this test thrice. Washed cells $\left(1 \times 10^{6}\right)$ were homogenized in assay buffer, resuspended in $500 \mu \mathrm{L}$ of PBS, and then lysed through ultrasonication. Assay and reaction buffers were added to $5 \mu \mathrm{L}$ of cell lysates and incubated for $15 \mathrm{~min}$ at $37^{\circ} \mathrm{C}$, and then $150 \mu \mathrm{L}$ of the color development reagent was added and mixed. Absorbance was measured at $520 \mathrm{~nm}$ using an iMark Microplate Reader.

\section{Tumorigenicity assay in nude mice}

Transfected cells were washed twice and resuspended in $1 \times$ Hank's buffer at a concentration of $5 \times 10^{6}$ cells/ $\mathrm{mL}$. A $100-\mu \mathrm{L}$ cell suspension was then injected subcutaneously into the left dorsal flank of 155 -week-old female nude mice; the right side was inoculated with SGC7901 cells transfected with vector alone and this served as the control. The larger $(a)$ and smaller $(b)$ tumor diameters were measured every week, and tumor volume was calculated as $a \times b^{2} \times 0.5$. At 3.5 weeks after injections, the mice were anesthetized with highconcentration diethyl ether until they died. Tumor specimens were split and collected. RT-PCR (described above) was used to analyze MAWD and MAWBP expression, and IHC analysis was used for detecting MAWBP, MAWD, TGF-beta, E-cadherin, and PGC protein expression. All animal procedures were approved by the Ethics Committee of the School of Oncology, Peking University (Beijing Cancer Hospital, China).

\section{Statistical analysis}

Statistical analyses were performed using Statistic Package for Social Science (SPSS) version 16.0. The $\chi^{2}$ test was used to define significant differences and univariate analysis among the pathological samples. $P<0.05$ was considered statistically significant. The Spearman rho test was performed to evaluate the protein correlations. The Kaplan-Meier method was used for predicting patient overall survival according to levels of MAWD and MAWBP expression. Student's $t$ test was used in measurement data.

\section{Results}

Characterization of MAWD and MAWBP coexpression and clinical outcome in gastric tumor

We compared the expression levels of MAWD and MAWBP proteins in the TMA that contained 223 GC samples and adjacent normal tissues. GC tissues showed faint or negative MAWD and MAWBP expression.
Representative IHC staining is shown in Fig. 1a. The rate of positive MAWD expression in gastric tumor tissues was $75 / 223(32.2 \%)$, which was lower than that in normal samples $(51 / 86 ; 59.3 \%)$ (Table 1). MAWBP showed the same expression pattern as MAWD did. The positivity rate of MAWBP in gastric tumor tissues was $62 / 223$ (26.6 \%), whereas it was 50/81 (61.7\%) in normal tissues (Table 1) (Fig. 1a). MAWD and MAWBP expression displayed statistically significant correlation $(P<0.001)$ (Table 2).

Further examination of the samples revealed that well-differentiated cancers tended to show uniform MAWD and MAWBP expression. The Kaplan-Meier survival curve indicated that prognosis was better for patients who expressed MAWD and MAWBP at high levels than for patients who expressed the proteins at low levels $(P<0.05)$ (Fig. 1b). These data suggest that analysis of the expression of both MAWD and MAWBP should provide useful information and might enhance the identification of differentiation grade and prognosis in patients.

\section{Correlation of TGF-beta, E-cadherin, and PGC protein expression with MAWD and MAWBP in GC tumors}

Given the clear relationship between MAWD and MAWBP expression and differentiation and the correlation of their expression with TGF-beta signaling, we performed TMA analysis for TGF-beta, E-cadherin, and PGC, which are GC differentiation-related proteins (Fig. 1c). The positive staining rates for these differentiation-related proteins in tumor and normal tissues were, respectively, the following (Table 1): TGF-beta, 105/223 (47.1\%) and 54/87 (62.1\%) $(P<0.05)$; E-cadherin, 95/223 (42.6\%) and 66/95 (69.5\%) $(P<0.001)$; and PGC, $86 / 223(38.6 \%)$ and $72 / 100(72 \%)$ $(P<0.001)$.

Relationship analysis revealed that MAWD and MAWBP expression was significantly correlated with the expression of TGF-beta $(P<0.001)$ and E-cadherin $(P<0.05)$ (Tables 3, 4). The results of Spearman rho test indicated the expression levels of MAWBP, MAWD, TGF-beta, and E-cadherin were correlated with each other $(P<0.05)$, and that the expression of E-cadherin was correlated with that of PGC $(P<0.001)$ (Table 5). Table 6 presents a summary of our analysis of patient clinicopathologic characteristics in relation to the expression level of each of the aforementioned proteins.

\section{Overexpression of MAWD and MAWBP in GC cells}

Previously, we detected endogenous expression of MAWD and MAWBP in GC cell lines using real-time PCR and western blotting. We found that MAWD and MAWBP are expressed at low levels in SGC7901 cells [21]. Thus, we selected SGC7901 as the test cell line and transfected the cells with the MAWD and MAWBP eukaryotic expression 


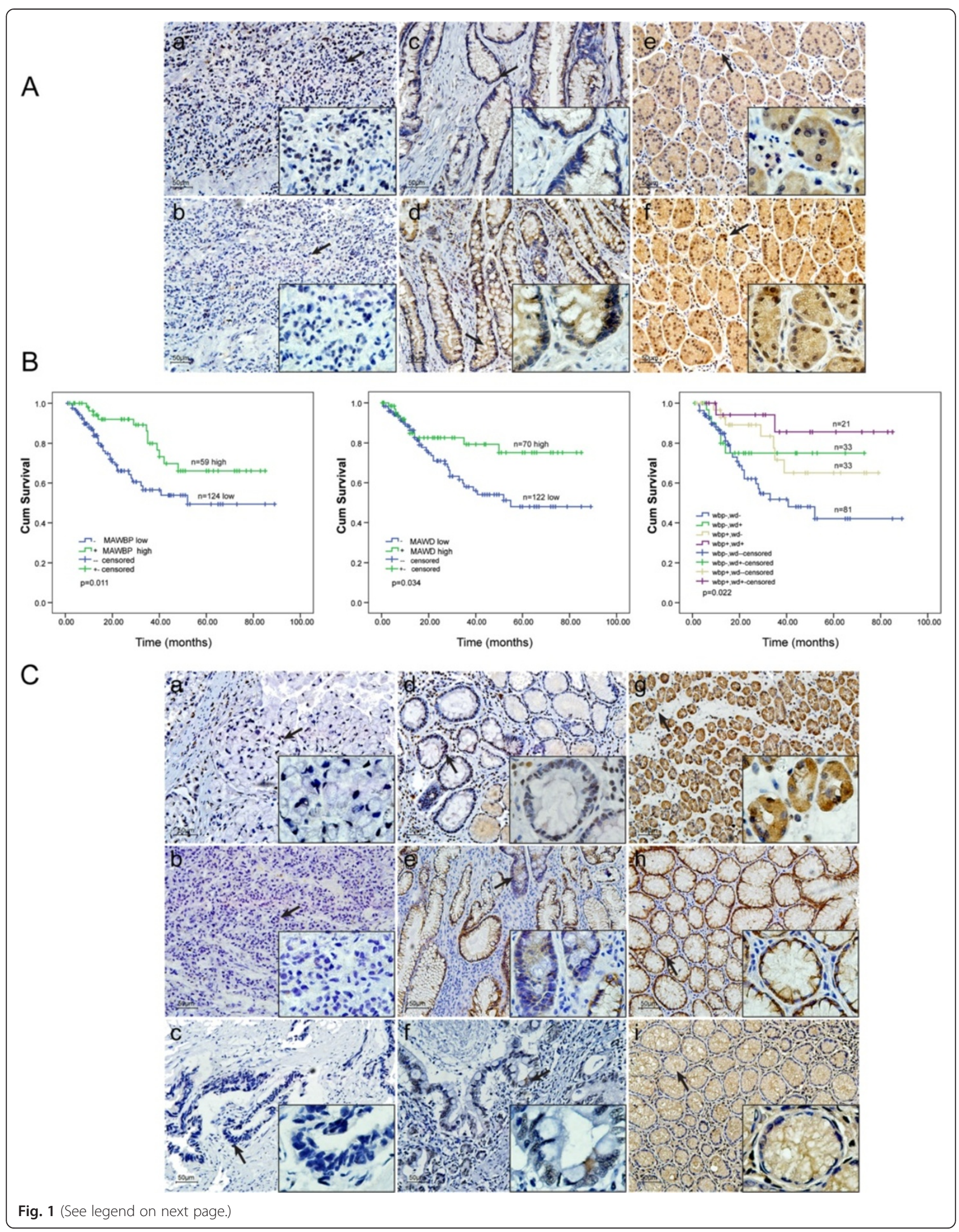


(See figure on previous page.)

Fig. 1 Comparison of MAWBP, MAWD, TGF-beta, E-cadherin, and PGC expression in GC and normal tissues by using IHC. (a) Comparison of MAWBP and MAWD expression in GC and normal tissues by means of TMA and IHC analysis (100x; 400x in the lower right corner). Weak MAWBP (a) and MAWD (b) protein staining in poorly differentiated carcinoma; expression of MAWBP (c) and MAWD (d) in intestinal metaplasia; strong positive staining of MAWBP (e) and MAWD ( $f$ ) in normal tissues $(P<0.001)$. (b) Kaplan-Meier analysis of overall survival in $G C$ patients expressing different levels of MAWBP and MAWD. (a) Green and blue lines represent the survival curves of patients expressing high and low levels of MAWBP ( $P$ $<0.05$ ). (b) Green and blue lines represent the survival curves of patients expressing MAWD at high and low levels $(P<0.05)$. (c) Combined MAWBP and MAWD expression for analysis of overall survival; prognosis was better for patients who expressed high levels of MAWBP and MAWD than for patients who expressed the proteins at low levels $(P<0.05)$. (c) Comparison of TGF-beta, E-cadherin, and PGC expression in GC and normal tissues by using TMA and IHC analysis (100x; 400x in the lower right corner). Weak TGF-beta (a), E-cadherin (b), and PGC (c) protein staining in poorly differentiated carcinoma; staining for TGF-beta (d), E-cadherin (e), and PGC (f) in intestinal metaplasia; strong positive staining for TGF-beta (g), E-cadherin (h), and PGC (i) in normal tissues $(P<0.05)$

vectors that we constructed; the cells were transfected with each of the vectors alone or with both vectors. We named these groups of cells MAWD (overexpressing MAWD alone), MAWBP (overexpressing MAWBP alone), MAW BP\&D (co-overexpressing MAWBP and MAWD), and Vector. Next, we isolated G418-resistant clones in order to obtain cells that stably overexpressed the proteins, and we used RT-PCR and western blotting to check for efficient expression of MAWD and MAWBP $(P<0.001$; Fig. $2 \mathrm{a}, \mathrm{b})$.

\section{MAWD and MAWBP coexpression induces differentiation in GC cells}

We performed western blotting, semiquantitative RTPCR, and confocal microscopy in order to examine the expression of the differentiation-related proteins E-cadherin, PGC, N-cadherin, and Snail in transfected cells. E-cadherin and PGC were used as differentiation markers of the gastric mucosa. The expression of Ecadherin protein and mRNA was increased relative to control in the MAWBP\&D group and was weakest in the Vector group (Fig. 3a, b), and this was also shown by the results of confocal microscopy and mean fluorescence-intensity measurements $(P<0.001$; Fig. $3 d)$. The expression of $\mathrm{N}$-cadherin was inversely associated with that of E-cadherin in the MAWBP\&D and Vector groups $(P<0.05$; Fig. 3a, b, d). However, the expression of PGC showed the same trend as E-cadherin expression: PGC expression was increased relative to control

Table 1 Comparison of MAWBP, MAWD, TGF-beta, E-cadherin, and PGC protein expression in GC and normal tissues

\begin{tabular}{llll}
\hline & Expression & & \\
\hline Protein & Tumor & Normal & P-value \\
& (\% positive) & (\% positive) & \\
MAWBP & $62 / 223(26.6)$ & $50 / 81(61.7)$ & $<0.001$ \\
MAWD & $75 / 223(32.2)$ & $51 / 86(59.3)$ & $<0.001$ \\
TGF-beta & $105 / 223(47.1)$ & $54 / 87(62.1)$ & 0.012 \\
E-cadherin & $95 / 223(42.6)$ & $66 / 95(69.5)$ & $<0.001$ \\
PGC & $86 / 223(38.6)$ & $72 / 100(72)$ & $<0.001$ \\
\hline
\end{tabular}

in the MAWBP\&D group and was lowest in the Vector group $(P<0.001$; Fig. 4$)$. Lastly, the expression of Snail protein was weakest in the MAWBP\&D group and increased in the Vector group $(P<0.05$; Fig. $4 \mathrm{a}, \mathrm{c})$. We found that cells in the MAWBP\&D group were well organized and appeared to exhibit polarity, whereas the cells in the control group were disorganized (Fig. 3d, Fig. 4c).

We also measured AKP activity to further analyze the differentiation level of various transfected cells. The AKP levels were the following (in U/g protein): MAWD group, $77.3 \pm 5.8$; MAWBP group, 74.8 \pm 3.9 ; MAWBP\&D group, $51.6 \pm 12.1$; and Vector group, $91.9 \pm 3.5$. AKP activity was lowest in the MAWBP\&D group and highest in the control group $(P<0.001$; Fig. 3c). Collectively, the aforementioned results suggest that MAWD and MAWBP induce the differentiation of GC cells.

\section{Potential MAWD and MAWBP protein-protein interaction (PPI) networks}

PPI networks were identified here and these provided complementary evidence to our previous proteomics studies on MAWD and MAWBP interactions. MAWD interacted with proteins related to the TGF-beta signaling pathway, including TGF-beta and Smad2 (Fig. 5a).

\section{Coexpression of MAWD and MAWBP influences the TGF-beta signaling pathway}

Western blotting analysis performed on the transfected cells revealed that p-Smad2 levels were lowest in the MAWBP\&D group and highest in the Vector group (Fig. 5b). Furthermore, the nuclear-translocation capacity of p-Smad2 was lowest in the MAWBP\&D group,

Table 2 Correlation of MAWBP and MAWD expression in GC

\begin{tabular}{lllll}
\hline \multirow{2}{*}{ Protein } & Expression & MAWBP & & \\
\cline { 3 - 5 } & & Positive (\%) & Negative (\%) & P-value \\
\hline MAWD & Positive & $32 / 215(14.9)$ & $42 / 215(19.5)$ & 0.001 \\
& Negative & $26 / 215(12.1)$ & $115 / 215(53.5)$ & \\
\hline
\end{tabular}


Table 3 Correlation of MAWD expression with TGF-beta, E-cadherin, and PGC expression in GC

\begin{tabular}{lllll}
\hline \multirow{2}{*}{ Protein } & Expression & MAWD & & \\
\cline { 3 - 5 } & & Positive (\%) & Negative (\%) & $P$-value \\
\hline TGF-beta & Positive & $49 / 209(23.4)$ & $52 / 209(24.9)$ & $<0.001$ \\
& Negative & $24 / 209(11.5)$ & $84 / 209(40.2)$ & \\
\multirow{2}{*}{ E-cadherin } & Positive & $37 / 200(18.5)$ & $49 / 208(23.6)$ & 0.039 \\
& Negative & $33 / 200(13.9)$ & $81 / 200(40.5)$ & \\
PGC & Positive & $35 / 218(16.5)$ & $48 / 218(22)$ & 0.45 \\
& Negative & $39 / 218(17.9)$ & $96 / 218(44)$ & \\
\hline
\end{tabular}

as shown by confocal microscopy (Fig. 5c), and the mean fluorescence intensity of $p-\operatorname{Smad} 2$ was highest in the Vector group $(P<0.05$; Fig. $5 \mathrm{~d})$. These results indicate that the MAWBP-MAWD complex could effectively suppress TGF-beta signaling by inhibiting downstream phosphorylation.

\section{Overexpression of MAWD and MAWBP affects the tumorigenicity of GC cells}

The results of in vivo experiments showed that tumor growth was slower in nude mice injected with cells of the MAWD, MAWBP, and MAWBP\&D groups than in mice injected with cells of the control group (Fig. 6a). Tumor growth was clearly slower after injection with cells of the MAWD and MAWBP groups as compared to that after injection of the Vector-group cells $(P<0.001$; Fig. $6 a)$. Moreover, the tumor volume in the MAWD and MAWBP overexpression groups was lower than that in the Vector group $(P<0.001$; Fig. $6 \mathrm{~b})$. RT-PCR results showed that MAWD and MAWBP were overexpressed in xenografts derived from cells transfected with MAWD and MAWBP (Fig. 6c), and this was confirmed by the immunostaining results (Fig. 6d). We also used IHC to evaluate the expression of TGF-beta, E-cadherin, and PGC in excised xenograft tumors; the proteins showed varied expression in distinct groups but the expression was higher in the MAWD and MAWBP overexpression groups than in

Table 4 Correlation of MAWBP expression with TGF-beta, E-cadherin, and PGC expression in GC

\begin{tabular}{lllll}
\hline \multirow{2}{*}{ Protein } & Expression & MAWBP & & \\
\cline { 3 - 5 } & & Positive (\%) & Negative (\%) & P-value \\
\hline TGF-beta & Positive & $43 / 216(19.9)$ & $59 / 216(27.3)$ & $<0.001$ \\
& Negative & $13 / 216(6.0)$ & $101 / 216(46.8)$ & \\
\multirow{2}{*}{ E-cadherin } & Positive & $31 / 208(14.9$ & $58 / 208(27.9)$ & 0.026 \\
& Negative & $25 / 208(15.7)$ & $94 / 208(45.2)$ & \\
PGC & Positive & $28 / 223(12.0)$ & $57 / 223(25.6)$ & 0.112 \\
& Negative & $32 / 223(14.3)$ & $106 / 223(47.5)$ & \\
\hline
\end{tabular}

Table 5 Correlations among the expression patterns of 5 proteins in GC

\begin{tabular}{llllll}
\hline Correlation coefficient $(\rho), N=223$ & & & \\
\hline & MAWBP & MAWD & TGF-beta & E-cadherin & PGC \\
MAWBP & 1 & $0.266^{* *}$ & $0.350^{* *}$ & $0.160^{*}$ & 0.107 \\
MAWD & 1 & $0.267^{* *}$ & $0.146^{*}$ & $0.136^{*}$ \\
TGF-beta & & 1 & $0.161^{*}$ & 0.048 \\
E-cadherin & & & 1 & $0.403^{* *}$ \\
PGC & & & & 1 \\
\hline${ }^{*} P<0.05,{ }^{* *} P<0.001$ & & & &
\end{tabular}

other groups (Fig. 6d). In Additional file 1, we present a model to illustrate the molecular functions of MAWD and MAWBP in the differentiation of GC cells.

\section{Discussion}

In this study, we systematically confirmed the correlation between the overexpression of MAWD and MAWBP and differentiation in GC tissues and cell lines. More importantly, we found that the coexpression of MAWD and MAWBP correlated with the expression of E-cadherin and PGC, which are differentiation-related factors in gastric cells. Furthermore, the expression of N-cadherin, Snail, and p-Smad2 was inversely associated with that of E-cadherin and PGC, and overexpression of MAWD and MAWBP reduced the nuclear translocation of Smad2 by attenuating its phosphorylation.

Previously, we reported proteomic data acquired from screening GC protein profiles, including those of MAWD and MAWBP, and we showed that these proteins can form a complex [8]. Thus, combined analysis of MAWD and MAWBP expression should provide useful information for uncovering the roles of these proteins in GC. We verified the expression of these 2 potential GC-related proteins in several GC tissue samples by means of TMA and IHC analyses. We found that MAWD and MAWBP were expressed at low levels in GC tissues, and that the expression of TGF-beta was also substantially decreased in GC; the expression levels of all 3 of these proteins were correlated. These results agree with previous observations. The proteins were also related to GC differentiation grade and patient prognosis. The survival times of patients who expressed high levels of MAWD and MAWBP were longer than those of patients who expressed these proteins at low levels.

Next, we analyzed the relationship between MAWD and MAWBP expression and differentiation in GC tissues by examining the differentiation-related proteins E-cadherin and PGC. E-cadherin plays a major role in cell-cell interactions, and a reduction in E-cadherin expression is correlated with de-differentiation, invasiveness, and 
Table 6 Univariate analysis with clinicopathological features in GC

\begin{tabular}{|c|c|c|c|c|c|}
\hline Features & MAWBP expression (\%) & MAWD expression (\%) & TGF-beta expression (\%) & E-cadherin expression (\%) & PGC expression (\%) \\
\hline \multicolumn{6}{|l|}{ Sex } \\
\hline Male & $37 / 151(24.5)$ & 44/146(30.1) & 69/149(46.3) & 60/143(41.9) & $54 / 152(35.5)$ \\
\hline Female & 22/71(30.9) & 25/68(36.8) & 29/65(44.6) & 35/64(54.6) & $36 / 72(50.0)$ \\
\hline$P$-value & 0.195 & 0.209 & 0.469 & 0.061 & 0.028 \\
\hline \multicolumn{6}{|c|}{ Age at diagnosis } \\
\hline$<60$ & 24/91(26.3) & $36 / 89(40.4)$ & 41/88(46.6) & 38/83(45.8) & 36/91(38.8) \\
\hline$\geq 60$ & $35 / 128(27.3)$ & $31 / 121(29.2)$ & $55 / 122(45.1)$ & 54/121(44.6) & $52 / 129(38.9)$ \\
\hline$P$-value & 0.500 & 0.017 & 0.469 & 0.492 & 0.512 \\
\hline \multicolumn{6}{|c|}{ TNM stage } \\
\hline I & $12 / 20(60.0)$ & $9 / 20(45.0)$ & $11 / 20(55.0)$ & $11 / 20(50.0)$ & 10/21(47.6) \\
\hline$\|$ & 18/78(17.2) & $24 / 75(32.0)$ & $31 / 75(41.3)$ & 28/72(38.9) & $32 / 78(41.0)$ \\
\hline III & $11 / 64(17.2)$ & $14 / 60(23.3)$ & 27/61(44.3) & $32 / 61(52.5)$ & 29/65(44.6) \\
\hline IV & 18/54(33.3) & $21 / 52(40.3)$ & $27 / 51(52.9)$ & 21/48(43.8) & 17/53(32.1) \\
\hline$P$-value & 0.001 & 0.160 & 0.501 & 0.355 & 0.481 \\
\hline \multicolumn{6}{|c|}{ Tumor depth } \\
\hline $\mathrm{T} 1-\mathrm{T} 2$ & 16/45(36) & 19/46(41.3) & 25/45(55.6) & $17 / 42(40.5)$ & 18/47(38.3) \\
\hline T3-T4 & 43/173(24.9) & 49/163(30.1) & 72/164(43.9) & 75/161(46.6) & 70/172(40.7) \\
\hline$P$-value & 0.107 & 0.105 & 0.111 & 0.298 & 0.451 \\
\hline \multicolumn{6}{|c|}{ Lymph node status } \\
\hline No & $21 / 52(40.4)$ & 20/52(38.5) & 27/51(52.9) & 24/47(51.1) & 23/54(42.6) \\
\hline N1 & 18/77(23.4) & 24/74(32.4) & $32 / 73(43.8)$ & 29/77(37.7) & $36 / 78(46.2)$ \\
\hline N3 & 12/68(17.6) & 18/62(29.0) & 29/66(43.9) & $31 / 62(50.0)$ & 23/66(34.8) \\
\hline N4 & $8 / 19(42.1)$ & 6/19(31.6) & 9/17(52.9) & 8/15(53.3) & 6/19(31.6) \\
\hline$P$-value & 0.016 & 0.760 & 0.681 & 0.339 & 0.452 \\
\hline \multicolumn{6}{|c|}{ Distant metastasis } \\
\hline MO & $51 / 190(26.8)$ & 56/183(30.6) & $81 / 182(44.5)$ & $82 / 179(45.8)$ & 78/192(40.6) \\
\hline M1 & 8/28(28.6) & $12 / 26(46.2)$ & $16 / 27(59.3)$ & 10/24(41.7) & 10/27(37.0) \\
\hline$P$-value & 0.504 & 0.089 & 0.110 & 0.437 & 0.446 \\
\hline \multicolumn{6}{|c|}{ Differentiation } \\
\hline D1 & $6 / 12(50.0)$ & 4/11(36.3) & 9/12(75.0) & $8 / 12(66.7)$ & 1/13(7.6) \\
\hline D2 & 30/78(38.5) & 28/76(36.8) & $41 / 74(55.4)$ & $36 / 71(50.7)$ & $32 / 79(40.5)$ \\
\hline D3 & 22/123(17.9) & $35 / 120(29.2)$ & 45/120(37.5) & $47 / 116(40.5)$ & $52 / 125(41.6)$ \\
\hline$P$-value & 0.031 & 0.649 & 0.006 & 0.046 & 0.035 \\
\hline
\end{tabular}

metastatic activity of carcinoma cells [22]. PGC is an aspartic protease produced mainly by the gastric mucosa [23], and the expression of PGC is used as a biomarker for the gastric mucosa. Moreover, a change in PGC expression might reflect gastric-cell differentiation [24], and the levels of E-cadherin and PGC can reflect the severity of gastric lesions or gastric-cell differentiation $[25,26]$. Here, we detected E-cadherin and PGC expression in the TMA, and we found that whereas the expression of MAWD, MAWBP, and TGF-beta was clearly correlated with that of E-cadherin, PGC expression was correlated with MAWD expression. These results provided evidence indicating that the expression of MAWD and MAWBP is closely related with the differentiation of GC.

We used PPI bioinformatic predictions to extract all available human proteins that are related to MAWD and MAWBP, and we described their global properties. PPI bioinformatic predictions could provide complementary evidence for genome-wide experimental 

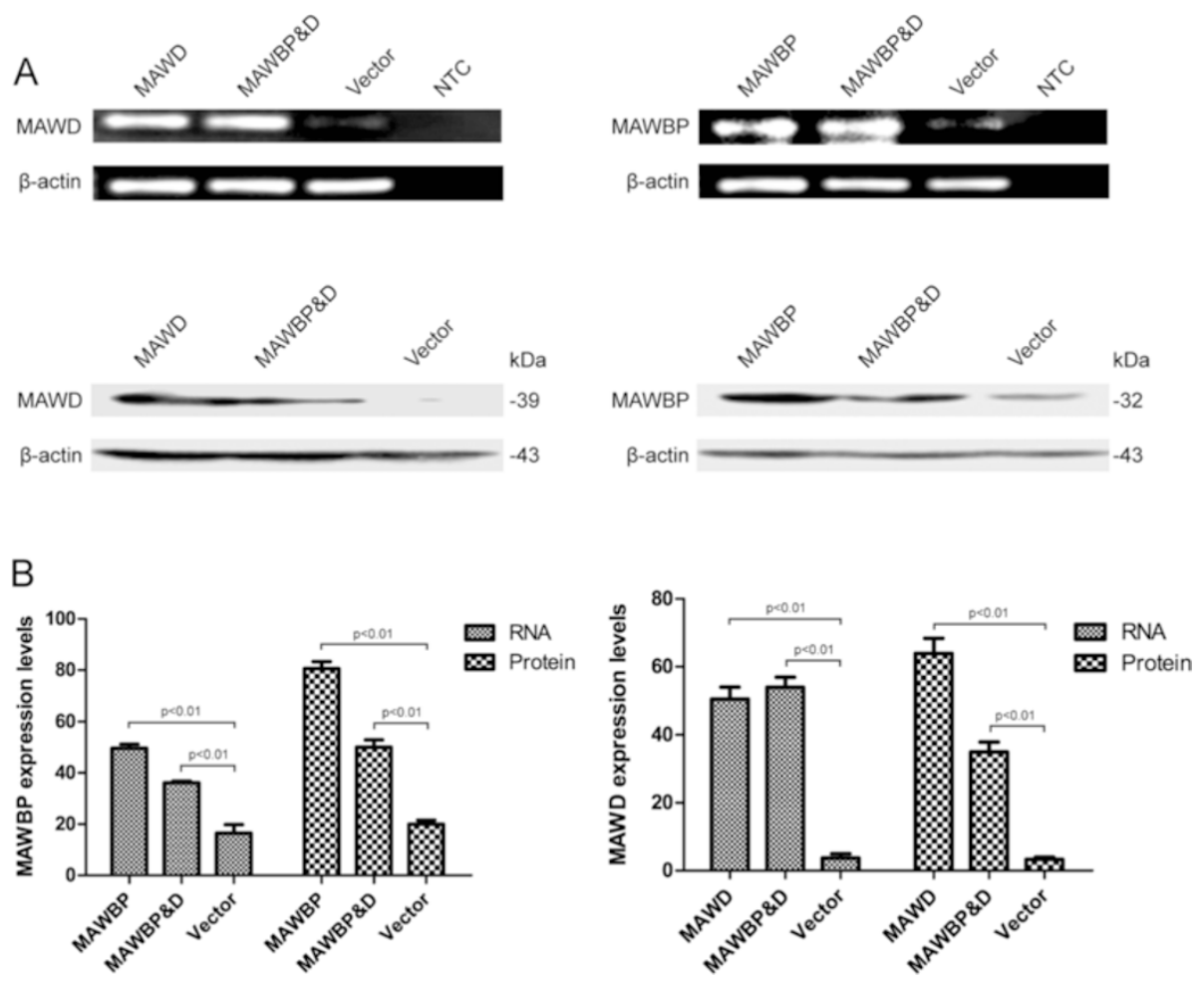

Fig. 2 Stable overexpression of MAWBP and MAWD in the GC cell line SGC7901. (a) Expression of MAWBP and MAWD was detected in stable clones by means of RT-PCR and western blotting. (b) The mRNA and protein levels of MAWBP and MAWD were higher in stable clones than in control cells $(P<0.001)$

studies. The function annotation of MAWD-interacting proteins indicated the potential involvement of MAWD and MAWBP in TGF-beta signaling.

We next evaluated the relationship between MAWD and MAWBP expression and differentiation in GC cells. We constructed eukaryotic expression vectors of MA WD and MAWBP, transfected them alone or together into SGC7901 cells, and examined the expression of the differentiation-related proteins E-cadherin and PGC in various transfected clones. We found that E-cadherin and PGC were strongly expressed in cells cotransfected with MAWBP and MAWD. Confocal analysis revealed that the cells in the MAWBP\&D group were well organized and appeared to exhibit polarity, whereas the cells in the control group were disorganized. Furthermore, the results of in vivo xperiments showed that tumor growth was slower in nude mice injected with cells of the MAWD, MAWBP, and MAWBP\&D groups as compared with that in mice injected with cells of the control group. E-cadherin and PGC were also expressed at the highest level in the xenograft tumors of the MAWBP\&D group. These results indicate that the cells in the MAWBP\&D group were differentiated to a greater extent than the cells in the other groups.
A malignant gastric tumor cell might also produce a particular isozyme of an enzyme, as illustrated most clearly in the case of AKP production. AKP activity was reported to be inversely proportional to GC cell differentiation [27]. We measured AKP activity in various transfected cells and found that the activity was lowest in the MAWBP\&Dtransfected clones, but highest in the Vector group. These results also suggested that the degree of differentiation was highest in the MAWBP\&D clones. Thus, overexpression of both MAWD and MAWBP induced GC differentiation.

E-cadherin is expressed by most epithelial tissues, and certain proteins expressed in cancer cells are also related to E-cadherin, such as Snail, Smad2, and Smad3. Conversely, $\mathrm{N}$-cadherin is an adhesion molecule that is typically expressed by mesenchymal cells. The loss of E-cadherin expression and the gain of $\mathrm{N}$-cadherin expression in cancer cells, occasionally referred to as "the cadherin switch," are functionally significant in cancer progression [28]. Furthermore, the molecule Snail could be related to E-cadherin because Snail can bind to specific DNA sequences called E-boxes present in the E-cadherin promoter and repress transcription [29]. Thus, we measured the expression levels of N-cadherin and Snail in the transfected GC cells; whereas E-cadherin was downregulated 


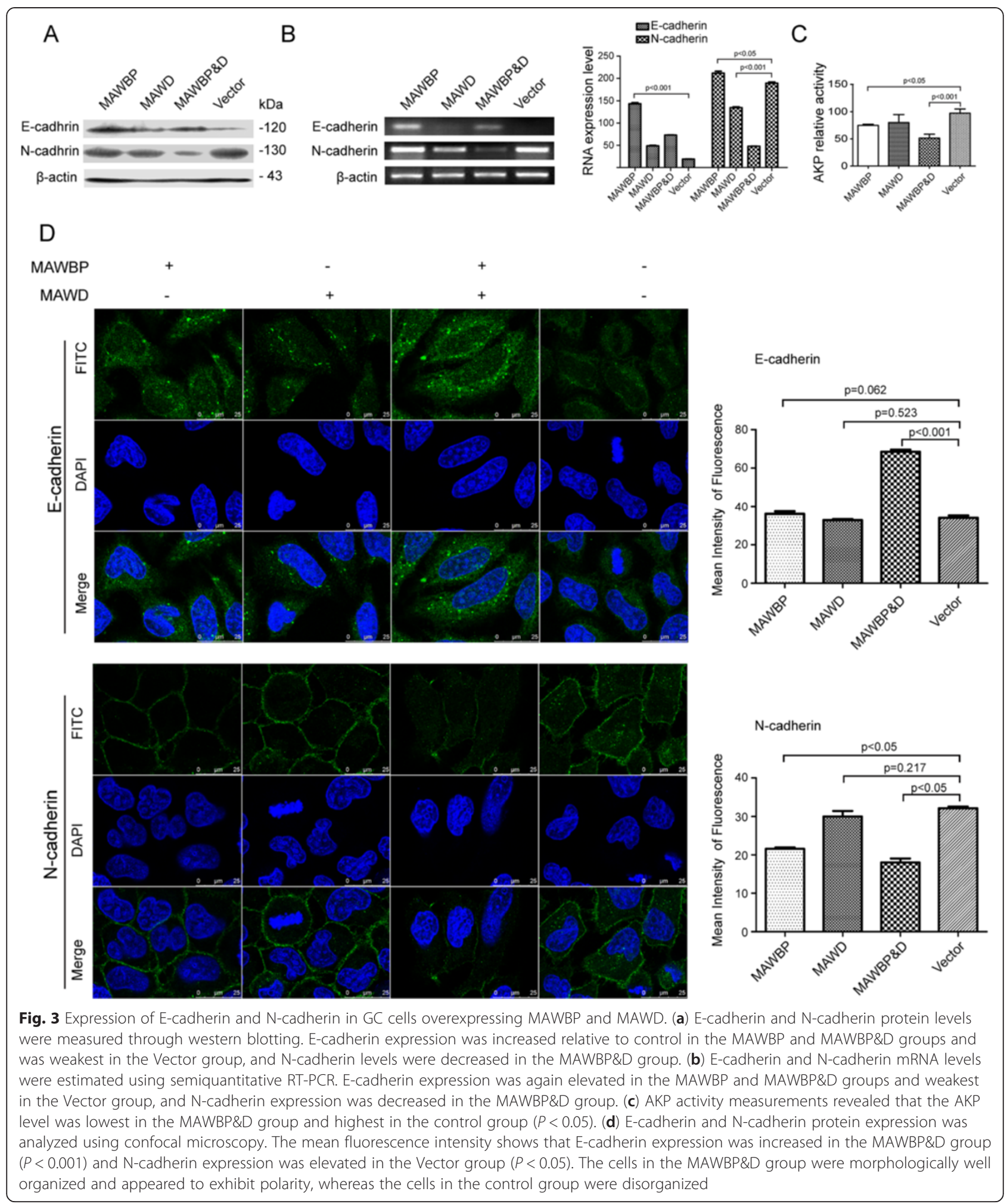

and N-cadherin was upregulated in control SGC7901 cells, E-cadherin was upregulated and $\mathrm{N}$-cadherin was downregulated in MAWBP\&D-cotransfected SGC7901 cells. Moreover, in MAWBP\&D-cotransfected cells, we also noted a reduction in the expression of Snail, a molecule that can be induced by TGF-beta stimulation 


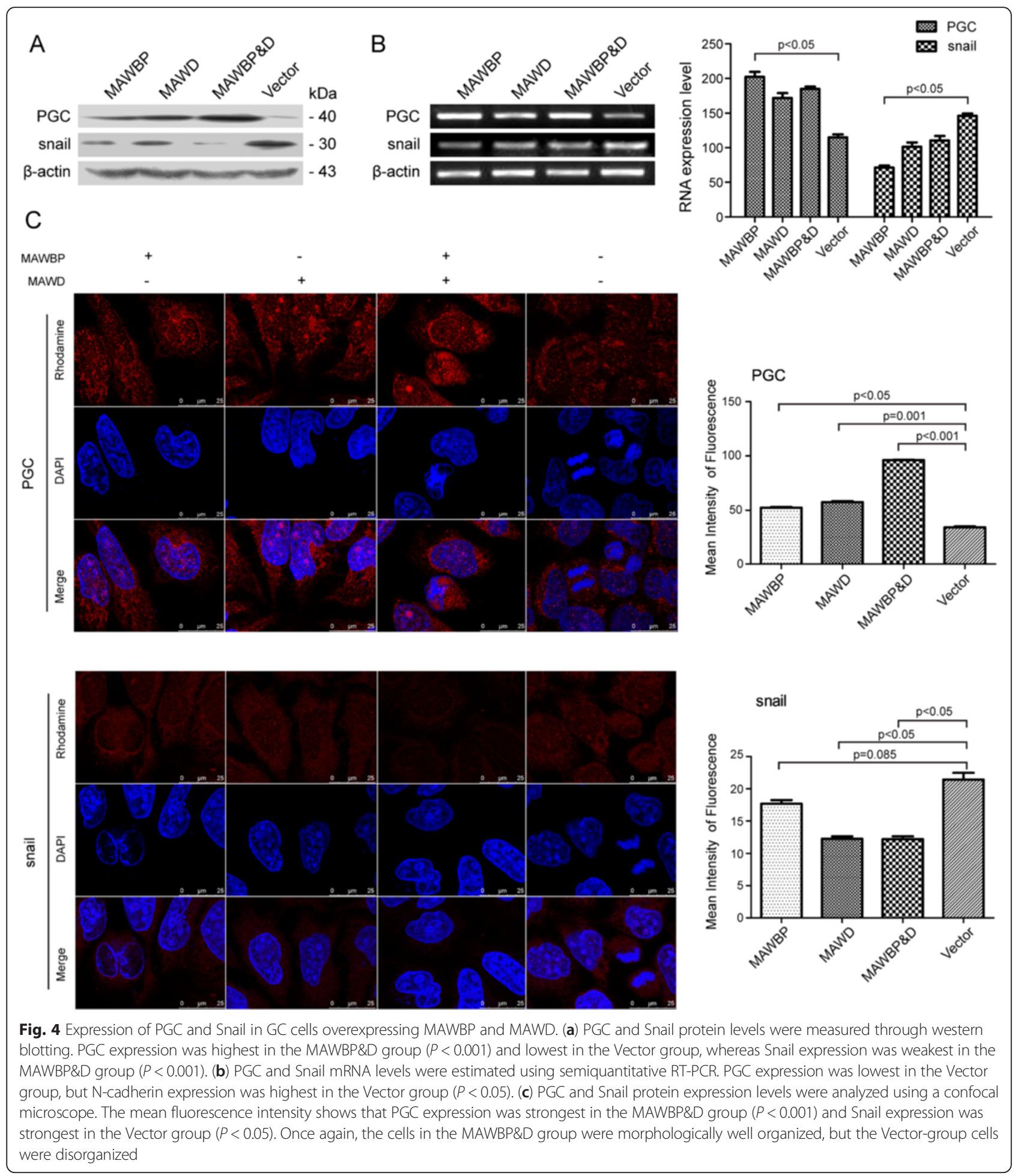

[30]. MAWD was found to recruit Smad7 and form a complex that inhibited TGF-beta signaling. Therefore, we evaluated TGF-beta activity in different transfected cells by examining the phosphorylation level and nuclear translocation of Smad2. We found that the
p-Smad2 level was lowest in the cells of the MAWBP\&D group but highest in the control-group cells, and that the nuclear-translocation ability of p-Smad2 was also weakest in the cotransfected cells. Our results suggest that MAWD cooperates with MAWBP to inhibit the 


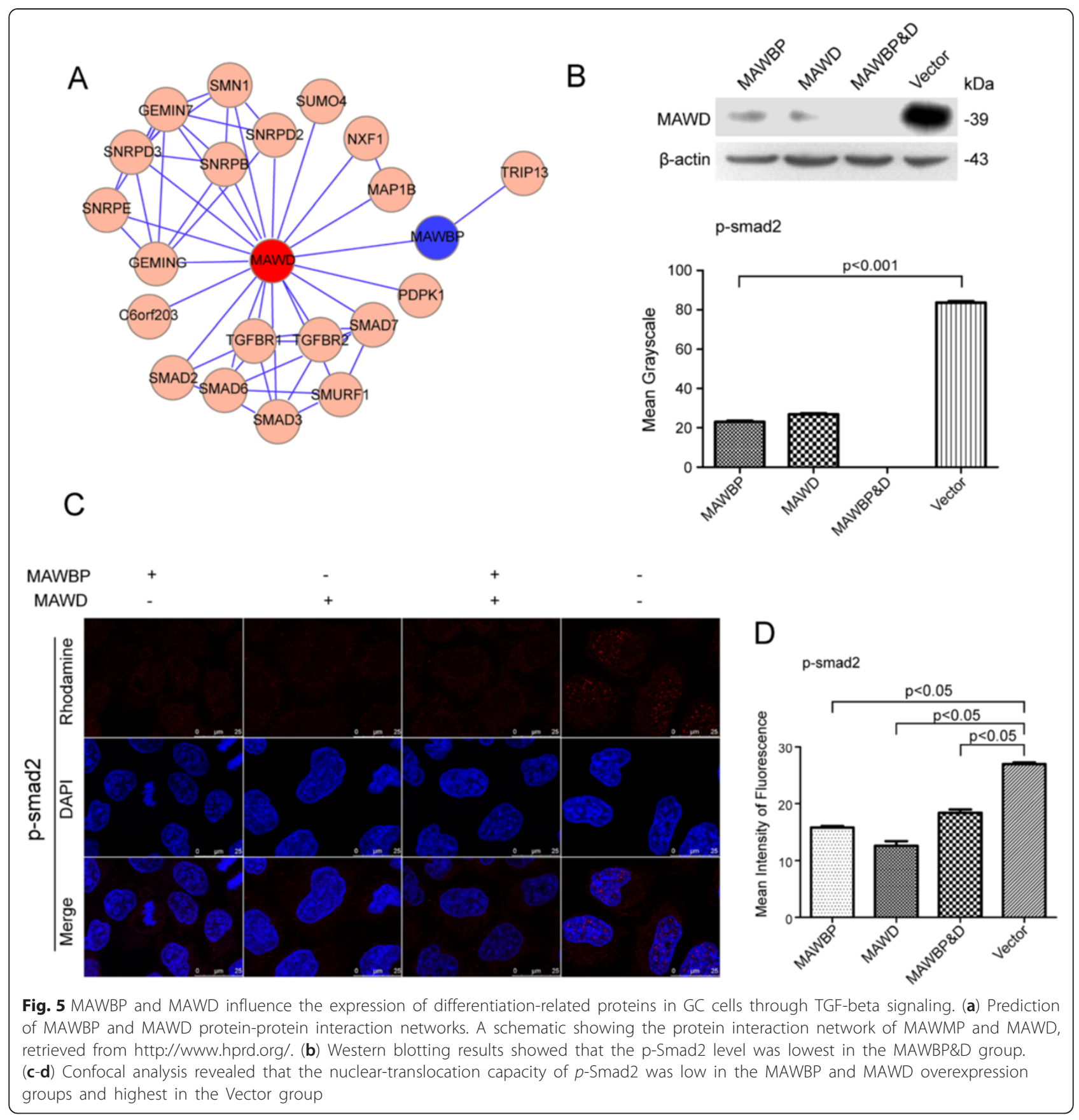

TGF-beta signaling pathway, which decreases Snail expression and increases E-cadherin expression; this, in turn, results in the well-differentiated characteristics of MAWBP\&D-cotransfected cells.

In conclusion, MAWD and MAWBP were downregulated in GC tissues and associated with the differentiation grade of these tissues. Low MAWD and MAWBP expression levels were associated with poor survival of patients. The results of in vitro and in vivo experiments demonstrated that the co-overexpression of MAWD and MAWBP induced GC differentiation. Lastly, detection of $\mathrm{p}$-Smad2 levels and its nuclear translocation revealed that MAWD and MAWBP induced the expression of differentiation-related proteins by modulating TGF-beta signaling in GC cells.

\section{Conclusions}

We discovered that MAWD and MAWBP, which were downregulated in GC tissues, were associated with differentiation in GC tissues. Coexpression of MAWD 


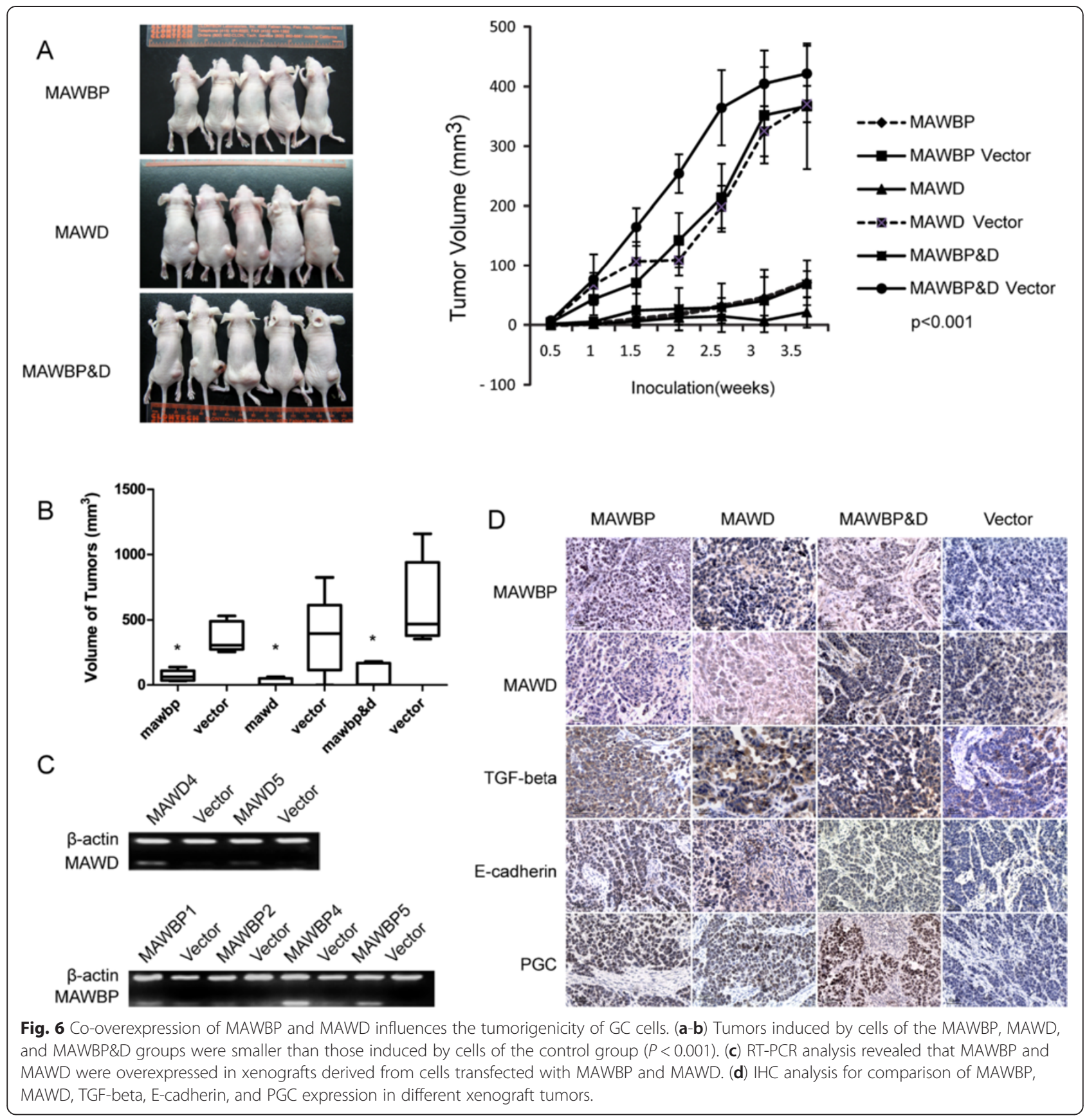

and MAWBP was correlated with the expression of the differentiation-related proteins E-cadherin and PGC in GC cells. The levels of Snail (a factor that negatively regulates $\mathrm{E}$-cadherin expression), $\mathrm{N}$-cadherin, and $\mathrm{p}$ Smad2 were inversely correlated with E-cadherin levels in cells that coexpressed MAWD and MAWBP. The results of AKP assays confirmed that these MAWD/MAWBPcoexpressing cells were differentiated to a greater extent as compared to control cells. Coexpression of MAWD and MAWBP also influenced the expression of E-cadherin and PGC in vivo. Our results suggest that the MAWD and
MAWBP might induce the expression of differentiationrelated proteins through the modulation of TGF-beta signaling in GC cells (Additional file 1).

\section{Additional file}

Additional file 1: Figure S1. A model illustrating the molecular functions of MAWBP and MAWD in GC. The presence of MAWBP enhances the inhibitory effect of MAWD on the TGF-beta signaling pathway. The MAWD-MAWBP complex inhibits the phosphorylation 
and nuclear translocation of Smads, which influences the expression of downstream genes, as shown by, for example, the downregulated expression of the transcription factor Snail. Snail does not efficiently bind to the E-box upstream of the E-cadherin gene, and thus the expression of E-cadherin is increased. This pathway influences the differentiation of GC cells. (TIFF $945 \mathrm{~kb}$ )

\section{Abbreviations}

MAWD: Mitogen-activated protein kinase activator with WD40 repeats; MAWBP: MAWD-binding protein; GC: Gastric cancer; TGF-beta: Transforming growth factor-beta; PGC: Pepsinogen C; TMA: Tissue microarray;

IHC: Immunohistochemistry; AKP: Alkaline phosphatase; EGFR: Epidermal growth factor receptor; PPI: Protein-protein interaction; RT-PCR: Reverse-transcription PCR: SPSS: Statistic package for social science.

\section{Competing interests}

The authors declare that they have no competing interests.

\section{Authors' contributions}

Dongmei Li conducted the experiments, analyzed the data, and drafted the manuscript. Jun Zhang and Youyong Lu designed the experiments and revised it critically for important intellectual content. Yu Xi and Lei Zhang helped with sample collection. Wenmei Li and Jiantao Cui were accountable for all aspects of the work in ensuring that questions related to the accuracy or integrity of all parts of the work were appropriately investigated and resolved. Rui Xing and Yuanmin Pan provided technical support and analyzed the data. Zemin Pan and Feng Li were responsible for bioinformatics analysis and literature search. All authors participated in writing the paper and approved the final version of the manuscript.

\section{Acknowledgements}

This study was supported by grants from the National Bio-Tech 863 Program (No.2006AA02A402), Natural Science Foundation of China (Grant: 30901717), and Basic Research for Application of Xinjiang (No.2015AG015). We thank the tissue bank of the Beijing Cancer Hospital \& Institute for providing gastric specimens.

\section{Author details}

'Department of Biochemistry and Molecular Biology, School of Medicine, Shihezi University, Xinjiang 832000, P.R China. ${ }^{2}$ Laboratory of Molecular Oncology, Key Laboratory of Carcinogenesis and Translational Research (Ministry of Education), Peking University Cancer Hospital/Institute, Beijing 100142, P.R China. ${ }^{3}$ Beijing Genomics Institute, Chinese Academy of Sciences, Shunyi, Beijing 101318, P.R China. ${ }^{4}$ Department of General Surgery, First Affiliated Hospital of Shihezi University, Shihezi, Xinjiang 832008, P.R China. ${ }^{5}$ Department of Laboratory, First Affiliated Hospital of Shihezi University, Shihezi, Xinjiang 832008 P.R, China.

\section{Received: 8 December 2014 Accepted: 1 September 2015}

\section{Published online: 15 September 2015}

\section{References}

1. Parkin DM, Bray F, Ferlay J, Pisani P. Global cancer statistics, 2002. CA Cancer J Clin. 2005;55(2):74-108.

2. Becker K, Langer R, Reim D, Novotny A, Meyer zum Buschenfelde C, Engel J. Significance of histopathological tumor regression after neoadjuvant chemotherapy in gastric adenocarcinomas: a summary of 480 cases. Ann Surg. 2011;253(5):934-9.

3. Hass HG, Smith U, Jager C, Schaffer M, Wellhauber U, Hehr T, et al. Signet ring cell carcinoma of the stomach is significantly associated with poor prognosis and diffuse gastric cancer (Lauren's): single-center experience of 160 cases. Onkologie. 2011;34(12):682-6.

4. Testino G. Gastric precancerous changes: carcinogenesis, clinical behaviour immunophenotype study and surveillance. Panminerva Med. 2006;48(2):109-18.

5. Sakamoto N, Oue N, Sentani K, Anami K, Uraoka N, Naito Y, et al. Liver-intestine cadherin induction by epidermal growth factor receptor is associated with intestinal differentiation of gastric cancer. Cancer Sci. 2012;103(9):1744-50.

6. Wei M, Wang Z, Yao H, Yang Z, Zhang Q, Liu B, et al. P27(Kip1), regulated by glycogen synthase kinase-3beta, results in HMBA-induced differentiation of human gastric cancer cells. BMC Cancer. 2011;11:109.
7. Hsu PI, Hsieh HL, Lee J, Lin LF, Chen HC, Lu PJ, et al. Loss of RUNX3 expression correlates with differentiation, nodal metastasis, and poor prognosis of gastric cancer. Ann Surg Oncol. 2009;16(6):1686-94.

8. Zhang J, Kang B, Tan X, Bai Z, Liang Y, Xing R, et al. Comparative analysis of the protein profiles from primary gastric tumors and their adjacent regions: MAWBP could be a new protein candidate involved in gastric cancer. J Proteome Res. 2007;6(11):4423-32.

9. Matsuda S, Katsumata R, Okuda T, Yamamoto T, Miyazaki K, Senga T, et al. Molecular cloning and characterization of human MAWD, a novel protein containing WD-40 repeats frequently overexpressed in breast cancer. Cancer Res. 2000;60(1):13-7.

10. Iriyama C, Matsuda S, Katsumata R, Hamaguchi M. Cloning and sequencing of a novel human gene which encodes a putative hydroxylase. J Hum Genet. 2001;46(5):289-92.

11. Buess M, Terracciano L, Reuter J, Ballabeni P, Boulay JL, Laffer U, et al. STRAP is a strong predictive marker of adjuvant chemotherapy benefit in colorectal cancer. Neoplasia. 2004;6(6):813-20.

12. Jung $\mathrm{H}$, Seong $H A$, Ha H. NM23-H1 tumor suppressor and its interacting partner STRAP activate p53 function. J Biol Chem. 2007;282(48):35293-307.

13. Dong JT. Chromosomal deletions and tumor suppressor genes in prostate cancer. Cancer Metastasis Rev. 2001;20(3-4):173-93.

14. Kim CJ, Choi BJ, Song JH, Park YK, Cho YG, Nam SW, et al. Overexpression of serine-threonine receptor kinase-associated protein in colorectal cancers. Pathol Int. 2007;57(4):178-82.

15. Halder SK, Anumanthan G, Maddula R, Mann J, Chytil A, Gonzalez AL, et al. Oncogenic function of a novel WD-domain protein, STRAP, in human carcinogenesis. Cancer Res. 2006;66(12):6156-66.

16. Hu L, Lu F, Wang Y, Liu Y, Liu D, Jiang Z, et al. RACK1, a novel hPER1interacting protein. J Mol Neurosci. 2006;29(1):55-63.

17. Datta PK, Moses HL. STRAP and Smad7 synergize in the inhibition of transforming growth factor beta signaling. Mol Cell Biol. 2000;20(9):3157-67.

18. Padua D, Massague J. Roles of TGFbeta in metastasis. Cell Res. 2009;19(1):89-102.

19. Kang B, Guo RF, Tan XH, Zhao M, Tang ZB, Lu YY. Expression status of ataxia-telangiectasia-mutated gene correlated with prognosis in advanced gastric cancer. Mutat Res. 2008;638(1-2):17-25.

20. Li Y, Lu YY. Isolation of diallyl trisulfide inducible differentially expressed genes in human gastric cancer cells by modified cDNA representational difference analysis. DNA Cell Biol. 2002;21(11):771-80.

21. DM, Zhang J, Li WM, Cui JT, Pan YM, Liu SQ, et al. MAWBP and MAWD inhibit proliferation and invasion in gastric cancer. World journal of gastroenterology : WJG. 2013;19(18):2781-92.

22. Maehata Y, Hirahashi M, Aishima S, Kishimoto J, Hirohashi S, Yao T, et al. Significance of dysadherin and E-cadherin expression in differentiated-type gastric carcinoma with submucosal invasion. Hum Pathol. 2011;42(4):558-67.

23. Okauchi Y, Iwahashi H, Okita K, Yuan M, Matsuda M, Tanaka T, et al. PGC1alpha Gly482Ser polymorphism is associated with the plasma adiponectin level in type 2 diabetic men. Endocr J. 2008:55(6):991-7.

24. He CY, Sun LP, Gong YH, Xu Q, Dong NN, Yuan Y. Serum pepsinogen II: a neglected but useful biomarker to differentiate between diseased and normal stomachs. J Gastroenterol Hepatol. 2011;26(6):1039-46.

25. Ning PF, Liu HJ, Yuan Y. Dynamic expression of pepsinogen $C$ in gastric cancer, precancerous lesions and Helicobacter pylori associated gastric diseases. World journal of gastroenterology : WJG. 2005;11(17):2545-8.

26. Kageyama T, Ichinose M, Tsukada-Kato $S$, Omata M, Narita Y, Moriyama A, et al. Molecular cloning of neonate/infant-specific pepsinogens from rat stomach mucosa and their expressional change during development. Biochem Biophys Res Commun. 2000;267(3):806-12.

27. Fishman WH. Recent developments in alkaline phosphatase research. Clin Chem. 1992;38(12):2484.

28. Araki K, Shimura T, Suzuki H, Tsutsumi S, Wada W, Yajima T, et al. E/N-cadherin switch mediates cancer progression via TGF-beta-induced epithelial-to-mesenchymal transition in extrahepatic cholangiocarcinoma. $\mathrm{Br}$ J Cancer. 2011;105(12):1885-93.

29. Li H, Wang H, Wang F, Gu Q, Xu X. Snail involves in the transforming growth factor beta1-mediated epithelial-mesenchymal transition of retinal pigment epithelial cells. PLoS One. 2011;6(8), e23322.

30. Vincent $T$, Neve EP, Johnson JR, Kukalev A, Rojo F, Albanell J, et al. A SNAIL1-SMAD3/4 transcriptional repressor complex promotes TGF-beta mediated epithelial-mesenchymal transition. Nat Cell Biol. 2009;11(8):943-50. 